\title{
Imaging the spin chirality of ferrimagnetic Néel skyrmions stabilized on topological antiferromagnetic $\mathrm{Mn}_{3} \mathrm{Sn}$
}

\author{
Teng Xu $\odot,{ }^{1,2, *}$ Zhen Chen $\odot,{ }^{3, *}$ Heng-An Zhou $\odot,{ }^{1,2}$ Zidong Wang $\odot,{ }^{1,2}$ Yiqing Dong, ${ }^{1,2}$ Lucia Aballe, ${ }^{4}$ Michael Foerster, ${ }^{4}$ \\ Pierluigi Gargiani, ${ }^{4}$ Manuel Valvidares, ${ }_{4}^{4}$ David M. Bracher, ${ }^{5}$ Tatiana Savchenko ${ }^{\circ},{ }^{5}$ Armin Kleibert $\odot,{ }^{5}$ Riccardo Tomasello, ${ }^{6}$ \\ Giovanni Finocchio, ${ }^{7}$ Soong-Guen Je, ${ }^{8}$ Mi-Young Im, ${ }^{8}$ David A. Muller, ${ }^{3,9, \dagger}$ and Wanjun Jiang ${ }^{1,2, \#}$ \\ ${ }^{1}$ State Key Laboratory of Low-Dimensional Quantum Physics and Department of Physics, and Tsinghua University, Beijing 100084, China \\ ${ }^{2}$ Frontier Science Center for Quantum Information, Tsinghua University, Beijing 100084, China \\ ${ }^{3}$ School of Applied and Engineering Physics, Cornell University, Ithaca, New York 14853, USA \\ ${ }^{4}$ ALBA Synchrotron Light Source, Cerdanyola del Vallès, 08290 Barcelona, Spain \\ ${ }^{5}$ Paul Scherrer Institut, 5232 Villigen PSI, Switzerland \\ ${ }^{6}$ Institute of Applied and Computational Mathematics, Foundation for Research and Technology—Hellas (FORTH), \\ GR-70013 Heraklion, Crete, Greece \\ ${ }^{7}$ Department of Mathematical and Computer Sciences, Physical Sciences and Earth Sciences, University of Messina, Messina 98166, Italy \\ ${ }^{8}$ Center for X-ray Optics, Materials Science Division, Lawrence Berkeley National Laboratory, Cyclotron Road, Berkeley, \\ California 94720, USA \\ ${ }^{9}$ Kavli Institute at Cornell for Nanoscale Science, Cornell University, Ithaca, New York 14853, USA
}

(Received 17 May 2021; revised 21 July 2021; accepted 26 July 2021; published 13 August 2021)

\begin{abstract}
Néel skyrmions are generally realized in asymmetric multilayers made of heavy metals (HMs) and ultrathin ferromagnets possessing strong interfacial Dzyaloshinskii-Moriya interactions (iDMIs). Depending on the relative strengths of iDMIs at the interfaces, various types of Néel skyrmions have been suggested, which are typified with characteristically different topological properties and current-driven dynamics. This suggests the importance of a precise quantification of their spin chiralities. In this paper, we explore the possibility of realizing Néel skyrmions in magnetic multilayers without the direct usage of standard HMs. Specifically, through depositing a thin layer of ferrimagnetic (FIM) CoTb layer on top of an antiferromagnetic (AFM) quantum material of composition $\mathrm{Mn}_{3} \mathrm{Sn}$, the AFM exchange interaction at the asymmetric interface provides an equivalent iDMI for stabilizing FIM Néel skyrmions. Secondly, through using advanced four-dimensional Lorentz scanning transmission electron microscopy (4D LSTEM), in combination with x-ray magnetic circular dichroism photoemission electron microscopy (XMCD-PEEM), we can directly determine the spin chirality of FIM Néel skyrmions. The present findings not only broaden the phase space for chiral interfacial magnetism but also provide a possibility for future applications of heavy-metal-free skyrmionic devices.
\end{abstract}

DOI: 10.1103/PhysRevMaterials.5.084406

\section{INTRODUCTION}

Magnetic skyrmions are noncollinear topological spin textures that have been extensively studied in bulk magnets [1-3] and magnetic multilayers [4,5]. For potential spintronic applications, interfacially asymmetric multilayers of stacking order heavy metal $l_{1} /$ ultrathin ferromagnet/heavy metal ${ }_{2}$ $\left(\mathrm{HM}_{1} / \mathrm{FM} / \mathrm{HM}_{2}\right)$, where $\mathrm{HM}_{2}$ can be either a different material or implying a different interface characteristic due to growth, are particularly promising for hosting roomtemperature Néel skyrmions [6-19]. In these multilayers, standard HM layers (such as Pt, Ta, W, Ir, and Pd) or topological insulators (TIs) with strong spin-orbit couplings (SOCs) are generally required for mediating the interfacial Dzyaloshinskii-Moriya interaction (iDMI) that subsequently determines the spin chirality of Néel-type spin textures

\footnotetext{
*These authors contributed equally to this article.

†David.a.muller@cornell.edu

¥jiang_lab@tsinghua.edu.cn
}

[20-28]. In this regard, it is important to explore alternative Néel-skyrmion-hosting multilayers without directly involving the standard HM layers. Investigations of Néel-type chiral spin textures stabilized on top of quantum materials with a relatively weak SOC, such as topological antiferromagnet $\mathrm{Mn}_{3} \mathrm{Sn}$ in this paper, could broaden the material choice of Néel skyrmions and greatly contribute to the current understanding of chiral interfacial magnetism [29,30]. Note that Bloch-type skyrmions have been observed in noncentrosymmetric bulk magnets such as $\mathrm{MnSi}$ and $\mathrm{FeGe}$, in which spins rotate spirally, which is different than the cycloidal rotation of spins in Néel-type skyrmions [31].

Another challenging topic is the direct imaging of the spin chirality of Néel skyrmions at room temperature. One of the most frequently used high-resolution techniques is Lorentz transmission electron microscopy (LTEM) $[3,13,32,33]$. It is worth mentioning that Néel skyrmions stabilized by iDMIs exhibit a uniform spin chirality [34,35], while Bloch-type magnetic bubbles stabilized by dipole interactions exhibit random spin topologies over the entire films [36,37]. It has been demonstrated that Néel skyrmions and Bloch-type bubbles 
show distinctively different contrasts at tilted conditions in LTEM images [38]. In perpendicularly magnetized multilayers, long-range dipolar interactions would preferentially form Bloch-type spin textures, while iDMIs would stabilize the Néel-type spin textures. Meanwhile, through detecting the tilt-dependent magnetic contrast, the LTEM technique can conclusively distinguish Néel from Bloch skyrmions [13,3941]. However, the in-plane components (spin chirality) of Néel skyrmions exhibit subtle contributions to the LTEM images, and their effects cannot be convincingly extracted $[13,32,33,39-41]$.

We will show here that the spin chirality of Néel skyrmions can be determined by using an advanced four-dimensional Lorentz scanning transmission electron microscopy (4D LSTEM), a determination which is not accessible in conventional LTEM. Our 4D LSTEM instrument is equipped with a high-dynamic-range electron microscopy pixel array detector (EMPAD) [40,42], which enables quantitative spin-texture imaging in embedded magnetic multilayers with a spatial resolution of a few nanometers toward atomic resolution. It is also capable of adding various external stimuli in the LSTEM setup, such as current, voltage, and magnetic field (along the beam direction) in a wide temperature range. It should be mentioned here that the chiral nature of ferrimagnetic (FIM) Néel skyrmions is, however, not directly revealed due to the technical limitations of different electron microscopes $[22,43-$ 46]. Additionally, a table for comparing different imaging techniques is given in Part 1 of the Supplemental Material [47]. Combined with $\mathrm{x}$-ray magnetic circular dichroism photoemission electron microscopy (XMCD-PEEM) imaging, we can further identify the antiparallel spin configurations of the sublattices of the FIM Néel skyrmion.

In this paper, we use one of the representative quantum materials, the noncollinear topological antiferromagnetic (AFM) $\mathrm{Mn}_{3} \mathrm{Sn}$ to replace the frequently utilized HM layers and to experimentally demonstrate the stabilization of Néel skyrmions at room temperature. $\mathrm{Mn}_{3} \mathrm{Sn}$ exhibits a noncollinear triangular AFM spin configuration with a vanishing net magnetic moment $[48,49]$, as shown in Fig. 1(a). Recently, a large anomalous Hall effect (AHE), a large anomalous Nernst effect, a large magnetic-optical effect, a magnetic spin Hall effect, and magnetic Weyl fermions have been observed in this noncollinear antiferromagnet [50-53]. These intriguing observations thus signify $\mathrm{Mn}_{3} \mathrm{Sn}$ as one of the representative quantum materials. Further, theoretical investigations have predicted a very weak SOC in the intrinsic noncollinear AFM $\mathrm{Mn}_{3} \mathrm{Sn}$ and their chiral magnetism [54-56]. Adjacent to magnetic layers (FIM CoTb in our case), interfacial chiral magnetism can be expected to be established at the surface of $\mathrm{Mn}_{3} \mathrm{Sn}$ through interlayer exchange coupling.

Interestingly, recent theoretical calculations have predicted that the triangular spin arrangement of the Mn sites possesses an iDMI of nonrelativistic origin [57]. Thus, the naturally existing iDMI in $\mathrm{Mn}_{3} \mathrm{Sn}$ could be harvested for stabilizing chiral spin structures in $\mathrm{Mn}_{3} \mathrm{Sn} / \mathrm{FM}$ bilayers. The feasibility of this proposal has been theoretically suggested through studying the interface between $\mathrm{L1}_{2}$-type $\mathrm{Mn}_{3} \mathrm{Ir}$ and $\mathrm{Co}$ (111), in which a substantial iDMI strength has been suggested through ab initio calculations [58]. Note that $\mathrm{L}_{2}$-type $\mathrm{Mn}_{3} \mathrm{Ir}$ and $\mathrm{Mn}_{3} \mathrm{Sn}$ are both noncollinear AFMs with the same triangular
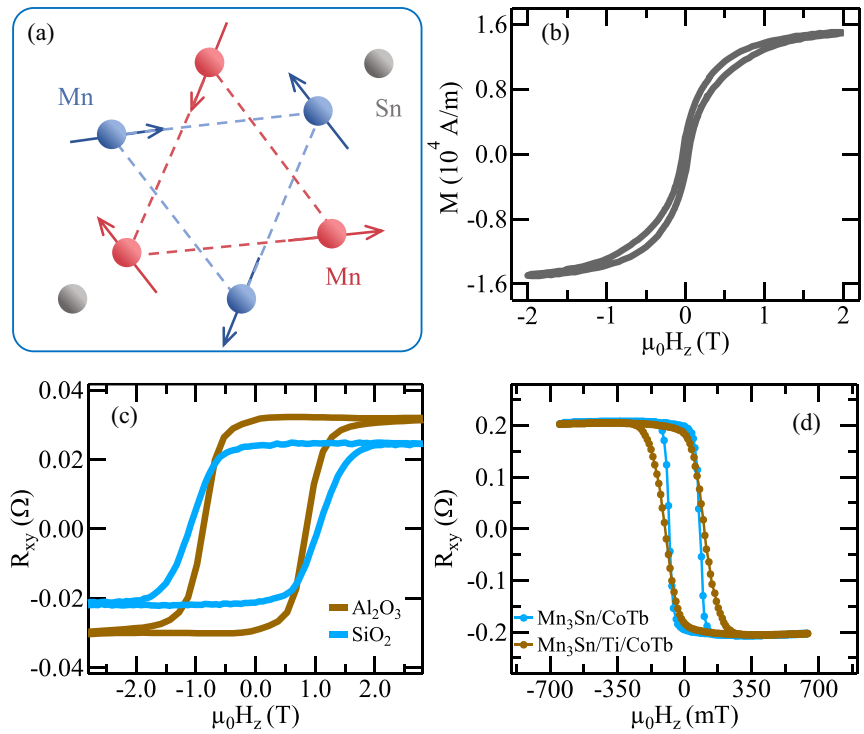

FIG. 1. Magnetic properties of the noncollinear antiferromagnetic (AFM) $\mathrm{Mn}_{3} \mathrm{Sn}$. (a) The triangular AFM spin lattices of $\mathrm{Mn}_{3} \mathrm{Sn}$. (b) The perpendicular magnetic hysteresis loop. (c) The room-temperature anomalous Hall effect (AHE) observed in $\mathrm{Mn}_{3} \mathrm{Sn}$ films grown on $\mathrm{Al}_{2} \mathrm{O}_{3}$ and $\mathrm{SiO}_{2}$ substrates following the same procedure. (d) The AHE loops measured in the $\mathrm{CoTb} / \mathrm{Mn}_{3} \mathrm{Sn}$ and $\mathrm{CoTb} / \mathrm{Ti} / \mathrm{Mn}_{3} \mathrm{Sn}$ multilayers at room temperature.

AFM spin arrangements [59]. Therefore, the iDMI with the same origin may also exist in the FIM/Mn ${ }_{3}$ Sn bilayer. This theoretical prediction partly motivates this paper.

\section{MATERIALS AND METHODS}

$\mathrm{Mn}_{3} \mathrm{Sn}$ thin films, possessing hexagonal $\mathrm{Ni}_{3} \mathrm{Sn}$-type structure with space group $P 63 / m m c$, were fabricated on the $\mathrm{Al}_{2} \mathrm{O}_{3}$ single crystalline and thermally oxidized silicon substrates by the AJA (Orion 8) ultrahigh vacuum magnetron sputtering system. Meanwhile, $\left[\mathrm{Mn}_{3} \mathrm{Sn}(3) / \mathrm{Co}_{75} \mathrm{~Tb}_{25}(6) / \mathrm{Si}_{3} \mathrm{~N}_{4}(2.9)\right]_{20}$ and $\left[\mathrm{Si}_{3} \mathrm{~N}_{4}(2.9) / \mathrm{Co}_{75} \mathrm{~Tb}_{25}(6) / \mathrm{Mn}_{3} \mathrm{Sn}(3)\right]_{20}$ (thickness in nanometers) magnetic multilayers were also deposited. The base pressure of the growth chamber was $2.0 \times 10^{-8}$ Torr, and the Ar working pressure was maintained at $3.0 \mathrm{mTorr}$ during film deposition. The deposition rate of $\mathrm{Mn}_{3} \mathrm{Sn}$ was $0.6 \AA / \mathrm{s}$. The 6-nm-thick CoTb layers with bulk perpendicular magnetic anisotropy (PMA) were prepared in a cosputtering mode, with their relative concentrations being adjusted by changing the relative growth powers. Magnetic multilayers were also deposited on the 100-nm- and 15-nm-thick $\mathrm{Si}_{3} \mathrm{~N}_{4}$ membranes for magnetic imaging using soft $\mathrm{x}$-ray microscopy (XM-1) and LTEM, respectively. The same multilayers were also grown on thermally oxidized silicon substrates for magnetic characterization and XMCD-PEEM imaging. The electronic transport properties of the samples were measured by using a physical property measurement system (PPMS, Quantum Design). The magnetic properties of the $\mathrm{Mn}_{3} \mathrm{Sn}$ films and multilayers were studied by using a superconductor quantum interference device (SQUID) magnetometer (MPMS, Quantum Design). The magnetic imaging was conducted at the Co $L_{3}$ edge $(778.5 \mathrm{eV})$ by using full-field magnetic transmission 
X-ray microscopy (MTXM) performed at the Advanced Light Source (ALS). The X-ray absorption spectroscopy (XAS) and $\mathrm{XMCD}$ measurements were conducted at the Co and $\mathrm{Mn}$ $L_{2,3}$ and the $\mathrm{Tb} M_{4,5}$ edges, respectively. The XMCD-PEEM experiments were performed at Paul Scherrer Institute (PSI) and ALBA Synchrotron Light Source.

\section{RESULTS AND DISCUSSION}

Figure 1(b) shows the room-temperature hysteresis loops $\left(M-H_{z}\right)$ measured with a perpendicular magnetic field $\left(H_{z}\right)$ for $\mathrm{Mn}_{3} \mathrm{Sn}$ films grown on top of [1102]-oriented $\mathrm{Al}_{2} \mathrm{O}_{3}$ substrate. A saturation magnetization $M_{\mathrm{s}}=1.2 \times 10^{4} \mathrm{~A} / \mathrm{m}$ is estimated, which is expected due to the noncollinear AFM order in $\mathrm{Mn}_{3} \mathrm{Sn}$. The $\mathrm{Mn}$ atoms form a slightly distorted kagome lattice, and the associated geometrical frustration manifests itself as an inverse triangular spin structure, which gives rise to weak magnetic moments [60,61]. The resulting small net magnetization thus allows the field-control of noncollinear antiferromagnetism in $\mathrm{Mn}_{3} \mathrm{Sn}$ and, more importantly, its electrical detection by the AHE [49]. Like the occurrence of the giant $\mathrm{AHE}$ in the $\mathrm{Mn}_{3} \mathrm{Sn}$ single crystal, a giant $\mathrm{AHE}$ is observed and shown in Fig. 1(c). Note that these samples were grown simultaneously on $\mathrm{Al}_{2} \mathrm{O}_{3}$ and $\mathrm{SiO}_{2}$ substrates. The occurrence of the giant AHE in both samples thus implies the presence of topological noncollinear spin configuration. Note that the amplitude of the AHE in $\mathrm{Mn}_{3} \mathrm{Sn} / \mathrm{SiO}_{2}$ is smaller than that of $\mathrm{Mn}_{3} \mathrm{Sn} / \mathrm{Al}_{2} \mathrm{O}_{3}$, indicating a smaller portion of $\mathrm{Mn}_{3} \mathrm{Sn}$ grains exhibit a triangular spin configuration. A 6-nm-thick $\mathrm{Co}_{75} \mathrm{~Tb}_{25}$ layer is subsequently deposited on top of $\mathrm{Mn}_{3} \mathrm{Sn}$. The presence of PMA is confirmed by the AHE measurement, which only probes the magnetization orientation of $\operatorname{Co}\left(M_{\mathrm{Co}}\right)$, because of the weak coupling between the conduction electron spins and magnetic moments of $\mathrm{Tb}\left(M_{\mathrm{Tb}}\right)$ [62]. The magnetization of $\mathrm{Tb}$ (parallel with external field) is opposite with that of Co (antiparallel with external field), due to the AFM coupling between the $\mathrm{Co}$ and $\mathrm{Tb}$ sublattices [62]. After inserting a thin layer of $\mathrm{Ti}(1 \mathrm{~nm})$ between $\mathrm{Mn}_{3} \mathrm{Sn}$ and $\mathrm{Co}_{75} \mathrm{~Tb}_{25}$, it is evident that the shape of the AHE loop is strongly modulated, as shown in Fig. 1(d). Together with the large coercive field and saturation field, the modulated AHE loops suggest the existence of an interlayer AFM exchange coupling between $\mathrm{Mn}_{3} \mathrm{Sn}$ and $\mathrm{Co}_{75} \mathrm{~Tb}_{25}$ films.

The opposite stacking order $\left[\mathrm{Mn}_{3} \mathrm{Sn} / \mathrm{Co}_{75} \mathrm{~Tb}_{25} / \mathrm{Si}_{3} \mathrm{~N}_{4}\right]_{20}$ and $\left[\mathrm{Si}_{3} \mathrm{~N}_{4} / \mathrm{Co}_{75} \mathrm{~Tb}_{25} / \mathrm{Mn}_{3} \mathrm{Sn}\right]_{20}$ is expected to provide a flipped sign for the iDMI and hence an opposite spin chirality, as schematically shown in Fig. 2(a). The layer-resolved film growth of multilayers and layer thickness can be determined from a cross-sectional STEM image, as shown in Fig. 2(b). Layered structures are further verified by the elemental mapping from the electron energy loss spectroscopy (EELS) for both films, as shown in Part 2 of the Supplemental Material [47]. Note that the $\mathrm{O} K$ edge overlaps with the $\mathrm{Sn} M$ edge, adding a small offset to the intensity of the Sn profile in EELS because of the inclusion of oxygen from the surface oxidation during TEM specimen preparation and should not be considered an intermixing between the Sn-containing and other layers. A $M-H_{z}$ loop measured with the perpendicular magnetic field $\left(H_{z}\right)$ is shown in Fig. 2(c), the shape of which is similar to typical Néel-skyrmion-hosting multilayers
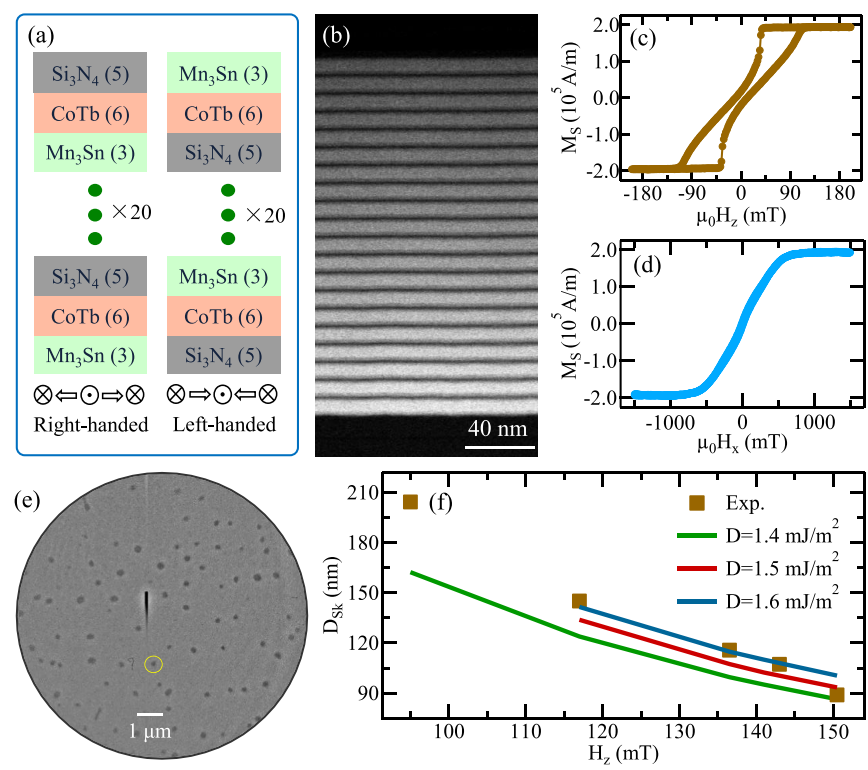

FIG. 2. Structures and magnetic properties of the interfacially asymmetric multilayers. (a) Schematic illustration of the asymmetric multilayers with an opposite stacking order. The numbers are the thickness of each layer in nanometers. (b) Cross-sectional scanning transmission electron microscopy (STEM) imaging of $\left[\mathrm{Mn}_{3} \mathrm{Sn} / \mathrm{Co}_{75} \mathrm{~Tb}_{25} / \mathrm{Si}_{3} \mathrm{~N}_{4}\right]_{20}$ multilayer. The scale bar is $40 \mathrm{~nm}$. (c) and (d) Out-of-plane and in-plane magnetic hysteresis loops for $\left[\mathrm{Mn}_{3} \mathrm{Sn} / \mathrm{Co}_{75} \mathrm{~Tb}_{25} / \mathrm{Si}_{3} \mathrm{~N}_{4}\right]_{20}$ multilayer. (e) The x-ray magnetic image at Co edge. (f) Comparison between the experimentally determined sizes of skyrmion (square dot), and results from micromagnetic simulations (line).

such as $[\mathrm{Pt} / \mathrm{CoFeB} / \mathrm{MgO}]_{15}$ and $[\mathrm{Pt} / \mathrm{Co} / \mathrm{Ir}]_{15}$, implying the possible formation of Néel skyrmions in our systems [7,8]. The (small) net saturation magnetization $M_{\mathrm{s}}=M_{\mathrm{Tb}}-M_{\mathrm{Co}}=$ $1.9 \times 10^{5} \mathrm{~A} / \mathrm{m}$ can be attributed to the partially compensated magnetism between the $\mathrm{Tb}$ and $\mathrm{Co}$ sublattices that gives rise to the formation of FIM Néel skyrmions [12]. Shown in Fig. 2(d) is the hysteresis loop measured in the sample plane $\left(H_{x}\right)$, from which the anisotropy field $H_{k} \approx 6000$ Oe can be estimated.

Magnetic imaging in the $\left[\mathrm{Mn}_{3} \mathrm{Sn} / \mathrm{Co}_{75} \mathrm{~Tb}_{25} / \mathrm{Si}_{3} \mathrm{~N}_{4}\right]_{20}$ multilayer was performed using soft $\mathrm{X}$-ray transmission microscopy (XM-1) with a spatial resolution down to $20 \mathrm{~nm}$ at the Co $L_{3}$ edge $(778.5 \mathrm{eV})$. Following the increase of $\mu_{0} H_{z}$, disordered labyrinthine domains gradually shrink into isolated bubblelike spin textures, which occurs from the competition between the exchange interaction, magnetic anisotropy, dipole-dipole interaction, and the (effective) iDMI, as confirmed by our micromagnetic simulations. A detailed examination of the magnetic domain configurations as a function of $\mu_{0} H_{z}$ is presented in Fig. S3 in Part 3 of the Supplemental Material [47]. Figure 2(e) shows a selected image acquired at $\mu_{0} H_{z}=141 \mathrm{mT}$, in which sparsely distributed bubblelike spin textures can be found. Through using a $360^{\circ}$ domain wall (DW) model [43], the diameter of these bubbles is determined to be in the range of 90-200 nm, which is larger than the resolution of the $\mathrm{x}$-ray transmission microscope $(20 \mathrm{~nm})$. Note that XM-1 probes only the out-of-plane 

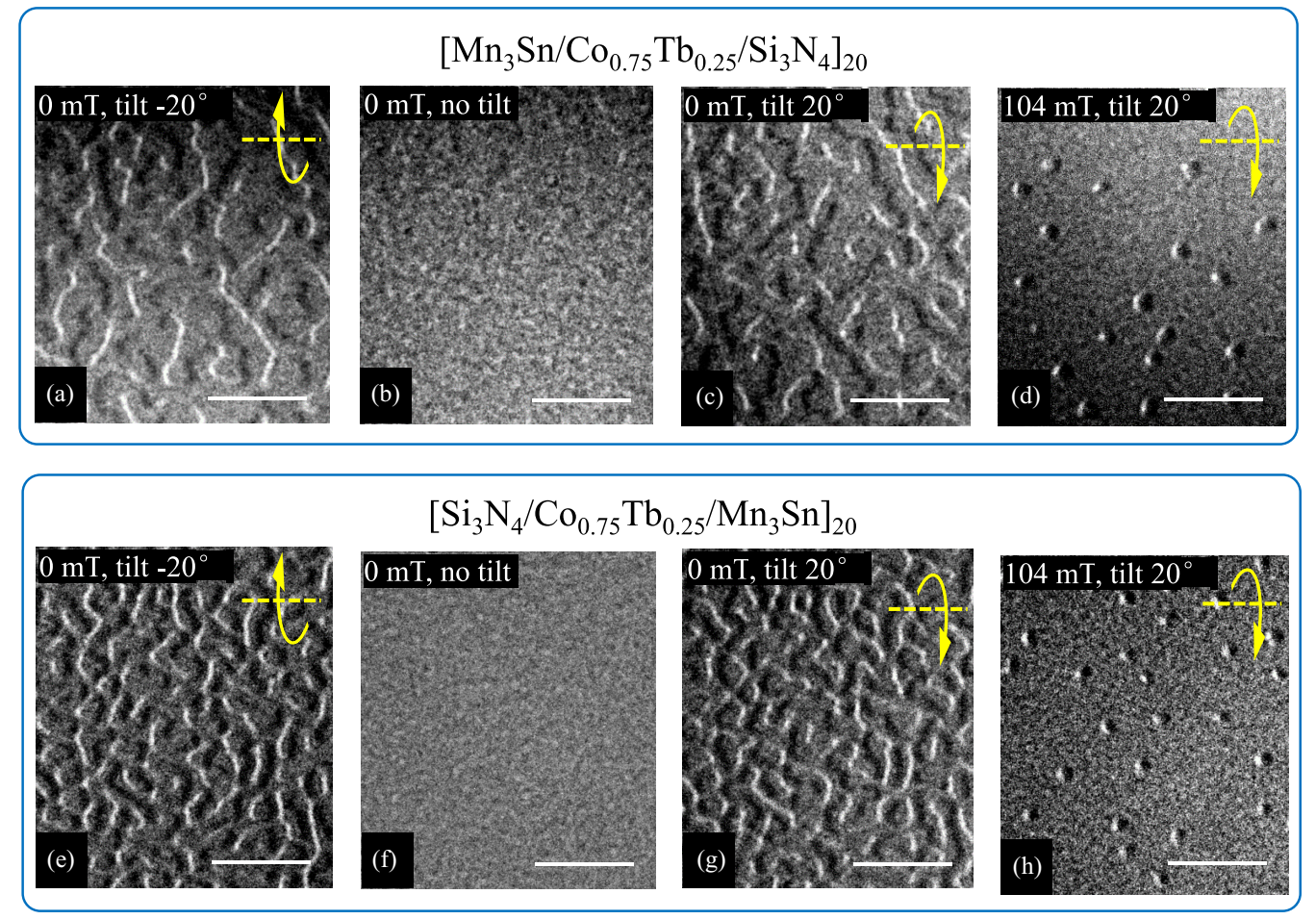

FIG. 3. Lorentz transmission electron microscopy (LTEM) images acquired at different magnetic fields and tilt angles. LTEM images for magnetic multilayer $\left[\mathrm{Mn}_{3} \mathrm{Sn} / \mathrm{Co}_{75} \mathrm{~Tb}_{25} / \mathrm{Si}_{3} \mathrm{~N}_{4}\right]_{20}$ at (a) $0 \mathrm{mT}$ and $-20^{\circ}$ tilt, (b) $0 \mathrm{mT}$ and $0^{\circ}$ tilt, (c) $0 \mathrm{mT}$ and $20^{\circ}$ tilt, and (d) $104 \mathrm{mT}$ and $20^{\circ}$ tilt. LTEM images for the inverted multilayer acquired at (e) $0 \mathrm{mT}$ and $-20^{\circ}$ tilt, (f) $0 \mathrm{mT}$ and $0^{\circ}$ tilt, (g) $0 \mathrm{mT}$ and $20^{\circ}$ tilt, and (h) $104 \mathrm{mT}$ and $20^{\circ}$ tilt. The scale bar is $1 \mu \mathrm{m}$.

magnetization component $\left(m_{z / \mathrm{Co}}\right)$ of the Co element because of the normal incidence of the soft $\mathrm{x}$ ray to specimens. The missing information on the orientation of the in-plane magnetization component $\left(m_{x, y / \mathrm{Co}}\right.$ and $\left.m_{x, y / \mathrm{Tb}}\right)$ is, however, extremely vital for quantifying the spin chirality of FIM Néel skyrmions, as shown below.

The size evolution of these bubblelike spin textures as a function of $\mu_{0} H_{z}$ is further summarized in Fig. 2(f). Following the increase of $\mu_{0} H_{z}$, the diameter of bubbles monotonically decreases. Based on the material-specific parameters, micromagnetic simulations were carried out using two coupled Landau-Lifshitz-Gilbert equations $[63,64]$. The $\mathrm{Co}_{75} \mathrm{~Tb}_{25}$ ferrimagnet with two sublattices composed of $\mathrm{Co}$ and $\mathrm{Tb}$ was considered. Note that a similar value of effective exchange $A=15 \mathrm{pJ} / \mathrm{m}$ for similar FIMs is reported in earlier works [62,65-67]. We analyzed a $500 \times 500 \mathrm{~nm}^{2}$ sample with a thickness of $6 \mathrm{~nm}$. A detailed description can be found in Part 4 of the Supplemental Material [47]. Using an effective iDMI parameter $D_{i}=1.5 \pm 0.1 \mathrm{~mJ} / \mathrm{m}^{2}$, the micromagnetic simulation largely reproduces the experimental data. This consistency has also been verified by multilayer simulations (20 repetitions) and thus suggests a semiquantitative estimation of a finite iDMI in the present multilayer.

Through detecting the phase shift of the electron beam induced by the lateral magnetic induction field $\left(B_{x, y}\right)$ in magnetic samples, LTEM has been frequently used to map out the profile of different types of spin textures $[3,32]$. In standard Lorentz mode with a normal incidence of electron beam, there is, however, no visible magnetic contrast for Néel-type spin textures, due to the absence of lateral magnetic induction. Tilting samples away from the normal incidence introduces magnetic contrasts, mainly originating from the lateral projection of the out-of-plane components of the magnetization $\left(m_{z}\right)[13,32,33]$. Such a tilt-dependent magnetic contrast has been widely used to distinguish Néel from Bloch DWs and skyrmions [13,39-41]. At zero field, overfocused LTEM images were acquired for the $\left[\mathrm{Mn}_{3} \mathrm{Sn} / \mathrm{Co}_{75} \mathrm{~Tb}_{25} / \mathrm{Si}_{3} \mathrm{~N}_{4}\right]_{20}$ multilayer, under a tilting angle from plane normal of $-20^{\circ}$, $0^{\circ}$, and $20^{\circ}$, as shown in Figs. 3(a)-3(c). In standard Lorentz mode, the external field was applied along the electron beam direction. Sparsely distributed bubblelike spin textures at 104 mT with a tilting angle $20^{\circ}$ are also shown in Fig. 3(d). Shown in Figs. 3(e)-3(h) are the corresponding LTEM images acquired in the inverted stack $\left[\mathrm{Si}_{3} \mathrm{~N}_{4} / \mathrm{Co}_{75} \mathrm{~Tb}_{25} / \mathrm{Mn}_{3} \mathrm{Sn}\right]_{20}$. At the zero-tilting angle, a diminishing magnetic contrast is evident in both samples. Through reversing the tilting angle from $-20^{\circ}$ to $20^{\circ}$, a reversal of magnetic contrast can also be seen. The reversal of bubblelike magnetic contrast at $104 \mathrm{mT}$, which is obtained at opposite tilting angles $\left(-20^{\circ}\right.$ and $\left.20^{\circ}\right)$, is also demonstrated in Part 5 of the Supplemental Material [47]. These results are consistent with the reported LTEM images of Néel-type spin textures. However, the in-plane components of the Néel-type spin textures $\left(m_{x, y}\right)$ have a subtle contribution to the LTEM images, and their effects cannot be extracted due to the challenges for quantifying the images [32,33]. Therefore, the spin chirality of these Néel skyrmions cannot be directly determined from the standard LTEM images [13,32,33,39-41]. 


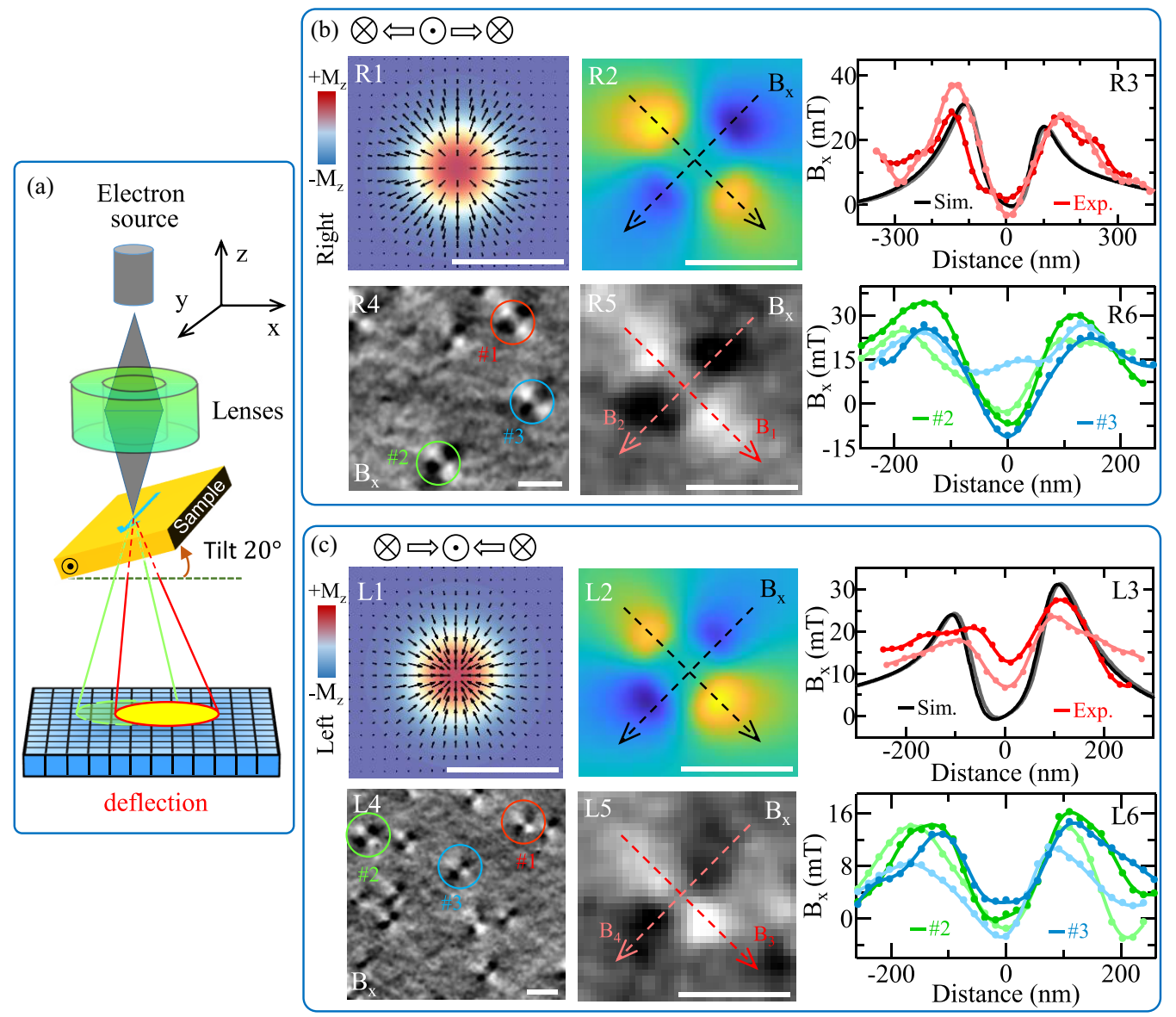

FIG. 4. Identifying the spin chirality of Néel skyrmions with four-dimensional Lorentz scanning transmission electron microscopy (4D LSTEM). (a) The schematic illustration of the 4D LSTEM setup. (b) and (c) Magnetic flux distributions for the right- and left-handed Néel skyrmions are shown in panels (R1) and (L1), while the horizontal component $\left(B_{x}\right)$ of the simulated magnetic flux distribution at $20^{\circ}$ tilt for the right- and left-handed Néel skyrmions are shown in panels (R2) and (L2), respectively. Experimentally determined magnetic fluxs of Néel skyrmions in $\left[\mathrm{Mn}_{3} \mathrm{Sn} / \mathrm{Co}_{75} \mathrm{~Tb}_{25} / \mathrm{Si}_{3} \mathrm{~N}_{4}\right]_{20}$ and $\left[\mathrm{Si}_{3} \mathrm{~N}_{4} / \mathrm{Co}_{75} \mathrm{~Tb}_{25} / \mathrm{Mn}_{3} \mathrm{Sn}\right]_{20}$ multilayers along the horizontal $(x)$ direction are shown in panels (R4) and (L4). Panels (R5) and (L5) are the enlarged images of the selected skyrmions (red circles) in panels (R4) and (L4). Panels (R3) and (L3) are the simulated (R2) and (L2) and experimental (R5) and (L5) line profiles of magnetic flux magnitude $\left(B_{x}\right)$ of the right- and left-handed Néel skyrmion along the direction marked with black and red arrows, respectively. Panels (R6) and (L6) are the experimental line profiles of the $B_{x}$ of a few undistorted skyrmions in panels (R4) and (L4), respectively. All the images are plotted under the same $x$ and $y$ axes in (a). The scale bars in (b) and (c) are $0.5 \mu \mathrm{m}$.

Differential phase contrast (DPC) imaging in LSTEM mode using a focused electron probe can directly measure the deflection angle of the electron beam $\left(\beta_{L}=\frac{e \lambda}{h} B_{x, y} t\right)$ induced by the Lorentz force of the lateral magnetic field $\left(B_{x, y}\right)$ in the sample with the possibility of reaching atomic resolution [68]. Here, $e$ is the unitary electronic charge, $\lambda$ is the wavelength of the electron beam, $h$ is the Planck constant, and $t$ is the thickness of the magnetic film. It provides the capabilities for quantitatively imaging the spin structures at a sub-5-nm spatial resolution, which are usually inaccessible from LTEM. The resolution of LTEM degrades to tens of nanometers due to the delocalization from the large defocus value required for gaining sufficient magnetic contrast [39], especially for compensated FIMs with a small saturation magnetization.

This is not a problem for LSTEM, which is operated in focus. High sensitivity to magnetic field from LSTEM relies on more illumination doses and therefore a high dynamic range detector. Figure 4(a) shows schematically our LSTEM setup equipped with a high-dynamic-range EMPAD. A 4D dataset is obtained by acquiring the full diffraction pattern at each scanning point. Compared with conventional DPC imaging, the 4D LSTEM setup can determine the deflection angle of the electron beam more accurately from the diffraction patterns via a more advanced analysis such as an edge detection algorithm [40]. These analyses can largely eliminate the artifacts from crystalline grains that are unavoidable in the sputtered polycrystalline or amorphous films [42]. Here, we show that 4D LSTEM can be utilized to directly visualize the spin chirality of the Néel skyrmions.

Employing a $360^{\circ}$ DW model [43] and following the established theory of Lorentz electron microscopy [69], we can simulate the magnetic induction field from Néel skyrmions with an opposite spin chirality. Panels (R2) and (L2) in Figs. 4(b) and 4(c) show simulated magnetic induction fields along the horizontal direction $\left(B_{x}\right)$ for a right-handed (R1) and a left-handed (L1) spin chirality of Néel skyrmions. The black curves in panels (R3) and (L3) show an asymmetric feature along the simulated diagonal direction $\left(B_{x}\right)$ for the 
right- and the left-handed spin chiralities, respectively. As illustrated from the separate contributions of the in-plane $\left(m_{x, y}\right)$ and out-of-plane $\left(m_{z}\right)$ magnetization components in Fig. S5 in Part 6 of the Supplemental Material [47], the reversal of the asymmetric intensity comes from the reversed spin orientation of the in-plane components $\left(m_{x, y}\right)$. This can thus be used to directly identify the spin chiralities of Néel skyrmions. Subtle asymmetric features can also be seen in the vertical component $\left(B_{y}\right)$ and the magnitude $\left(|\mathbf{B}|=\sqrt{B_{x}^{2}+B_{y}^{2}}\right)$, as shown in Fig. S6 in Part 7 of the Supplemental Material [47]. Our quantitatively calculated magnetic induction fields thus suggest that 4D LSTEM can be used for mapping out the spin chirality of Néel skyrmions.

Panels (R4) and (L4) show the experimentally acquired magnetic flux $\left(B_{x}\right)$ images using 4D LSTEM. The selected right- and left-handed Néel skyrmions [red circles in panels (R4) and (L4)] are shown in the panels (R5) and (L5), which were acquired from the $\left[\mathrm{Mn}_{3} \mathrm{Sn} / \mathrm{Co}_{75} \mathrm{~Tb}_{25} / \mathrm{Si}_{3} \mathrm{~N}_{4}\right]_{20}$ and $\left[\mathrm{Si}_{3} \mathrm{~N}_{4} / \mathrm{Co}_{75} \mathrm{~Tb}_{25} / \mathrm{Mn}_{3} \mathrm{Sn}\right]_{20}$ multilayers with flipped surfaces, respectively. The red curves in panels (R3) and (L3) show their line profiles along the diagonal directions of experimental magnetic flux $B_{x}$. The asymmetric features qualitatively agree with the simulated results, as shown in panels (R3) and (L3). A small residual offset in the zero point of the magnetic field may exist from LSTEM measurements because the zero-field point was calibrated by zeroing the beam deflection angle from sample regions far from skyrmions and without apparent magnetic contrast. The possible long-range field from skyrmions can induce a small shift in the magnetic field. The overall shape of the vertical direction $\left(B_{y}\right)$ and magnitude $(|B|)$ of the magnetic induction field images also qualitatively agree with the simulations, as shown in Part 8 of the Supplemental Material [47]. Thus, the spin chirality of Néel skyrmions, being the right- or left-handed in these two inverted multilayers, was confirmed. Note that the rightand left-handed spin chiralities correspond to positive and negative signs of the iDMI parameters, respectively, which is expected for the inverted stacking orders [70]. We also find that local sample bending or crystalline grains may introduce artifacts into the magnetic flux images, which were carefully avoided during our experiments. Furthermore, the signal-to-noise ratio of the magnetic field images scales with the exposure dose, requiring a high dynamic range detector like the EMPAD for identifying the spin chirality of Néel skyrmions $[42,71]$.

We also note that the shape or size of magnetic flux distribution from Néel skyrmions can be affected by many factors, such as local defects pinning or residual magnetic field from neighboring Néel skyrmions. In our experiments, we chose Néel skyrmions without apparent distortions. Line profiles of magnetic field from both $B_{x}$ and $B_{y}$ components of more undistorted Néel skyrmions are further shown in panels (R6) and (L6) in Figs. 4 and S8 in Part 9 of the Supplemental Material [47], respectively. It is worth mentioning that the small in-plane external magnetic field $(<50 \mathrm{mT})$ is much smaller than those of the effective field of the iDMI and should have negligible effects on the identification of the chirality in the films. Note that the 4D LSTEM experiment was also performed in $\left[\mathrm{Si}_{3} \mathrm{~N}_{4} / \mathrm{Co}_{75} \mathrm{~Tb}_{25}\right]_{20}$ multilayers, in which the
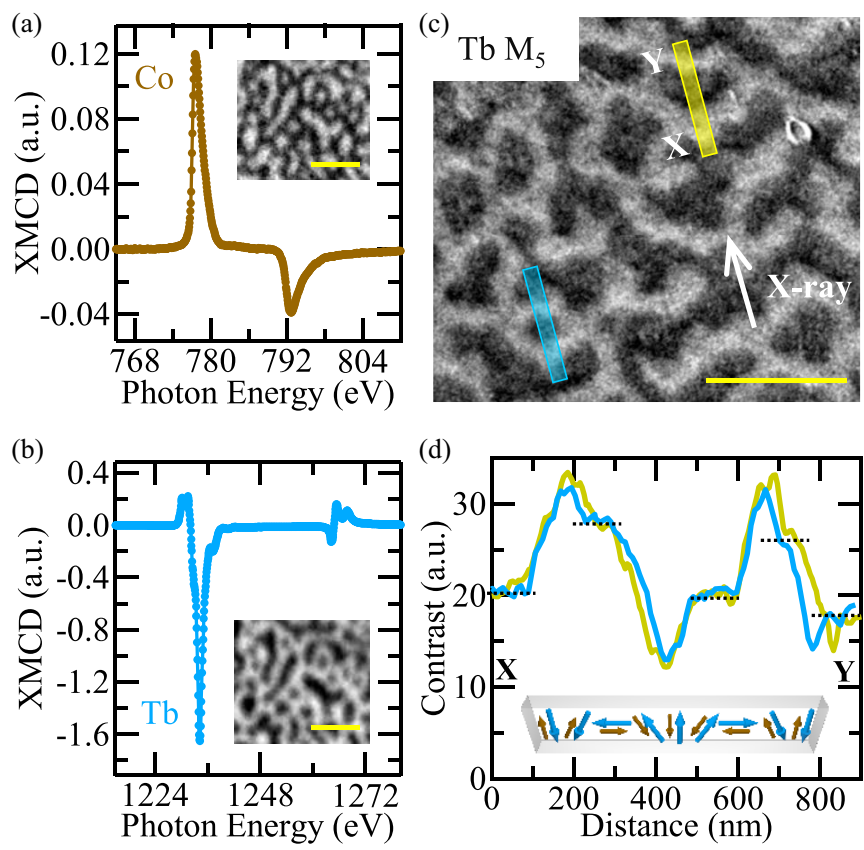

FIG. 5. Chirality determination of ferrimagnetic (FIM) Néel skyrmions using $\mathrm{x}$-ray magnetic circular dichroism photoemission electron microscopy (XMCD-PEEM). (a) and (b) The XMCD spectra measured at $\mathrm{Co} L_{3}$ edge and $\mathrm{Tb} M_{5}$ edge in the $\left[\mathrm{Mn}_{3} \mathrm{Sn} / \mathrm{Co}_{75} \mathrm{~Tb}_{25} / \mathrm{Si}_{3} \mathrm{~N}_{4}\right]_{20}$ multilayer at $\mu_{0} H_{z}=+2$ Tesla, respectively. Insets in (a) and (b) are the corresponding XMCD-PEEM images acquired at zero magnetic field. Scale bar is $1 \mu \mathrm{m}$. (c) XMCD-PEEM image with an enhanced contrast at the $\mathrm{Tb} M_{5}$ edge by averaging 500 images. The white arrow indicates the incident direction of the $\mathrm{x}$ ray. Scale bar is $1 \mu \mathrm{m}$. (d) Laterally averaged linescans of the magnetic contrast from the position marked locations in (c). Dash lines indicate the XMCD brightness level of the corresponding out-of-plane domains, while additional dips/peaks between them correspond to in-plane magnetic moment of the chiral domain walls (DWs). Inset is the schematic of the chiral spin texture. The blue color represents $\mathrm{Tb}$, while dark yellow corresponds to $\mathrm{Co}$ sublattices.

presence of Bloch-type spin textures was identified, as illustrated in Part 10 of the Supplemental Material [47]. We also notice that Bloch-type bubbles with random spin topology were recently reported in the very thick CoTb film without an interfacial asymmetry [72]. These results further suggest the important role of noncollinear $\mathrm{AFM} \mathrm{Mn}_{3} \mathrm{Sn}$ in introducing interfacial chiral magnetism.

The incorporation of the FIM $\mathrm{Co}_{75} \mathrm{~Tb}_{25}$ layer with two AFM-coupled $\mathrm{Tb}$ and $\mathrm{Co}$ sublattices results in the formation of FIM Néel skyrmions. The 4D LSTEM measurement is, however, only sensitive to the net magnetization $\left(\left|M_{\mathrm{Tb}}-M_{\mathrm{Co}}\right|\right)$. To confirm the presence of FIM Néel skyrmions and their associated (opposite) spin chiralities in an element-specific measurement, we also performed XMCD spectroscopy and XMCD-PEEM experiments, as shown in Fig. 5. Measuring Co $L_{2,3}$ and $\mathrm{Tb} M_{4,5}$ absorption edges with XMCD spectra at $\mu_{0} H_{z}=2 \mathrm{~T}$, opposite signs are evident, corresponding to the antiparallel magnetization of Co and $\mathrm{Tb}$ atoms. Note that the vanishing XMCD signal at $\mathrm{Mn}$ $L_{2,3}$ absorption edge can be observed, which excludes the 
proximity-induced magnetism in $\mathrm{Mn}_{3} \mathrm{Sn}$, as shown in Fig. S10 in Part 11 of the Supplemental Material [47]. Insets of Figs. 5(a) and 5(b) show XMCD-PEEM images of an identical area acquired at the $\mathrm{Co} L_{3}$ and $\mathrm{Tb} M_{5}$ edges at zero magnetic field, respectively. The reversed magnetic contrasts between these two images further confirm the AFM coupling between the $\mathrm{Tb}$ and Co sublattices.

In the XCMD-PEEM investigations, the samples are illuminated with the $\mathrm{x}$-ray beam at a small grazing angle (typically $16^{\circ}$ ) with respect to the film plane. Hence, the magnetic contrast of the in-plane magnetization component $\left(m_{x, y}\right)$ is $\sim 3.5$ times stronger than that of the out-of-plane components $\left(m_{z}\right)$ [24]. Specifically, the change of magnetic contrast correlates with the relative orientation between the in-plane magnetization and the $\mathrm{x}$-ray incidence direction. The contrast is bright when the in-plane magnetization is antiparallel with the $\mathrm{x}$-ray incidence direction, whereas a dark contrast is present when they are in a parallel configuration. Thus, together with its high spatial resolution (possibly down to $25 \mathrm{~nm}$ ), XMCD-PEEM can be independently employed for confirming the spin chirality of Néel-type spin textures.

Figure 5(c) displays a high-resolution XMCD-PEEM image of the multidomain states at zero field for the $\left[\mathrm{Mn}_{3} \mathrm{Sn} / \mathrm{Co}_{75} \mathrm{~Tb}_{25} / \mathrm{Si}_{3} \mathrm{~N}_{4}\right]_{20}$ multilayer acquired at the $\mathrm{Tb}$ $M_{5}$ edge. Along the x-ray illumination direction, an alternating contrast change from brighter to darker can be observed, which corresponds to the down-to-up $(\downarrow \swarrow \leftarrow \nwarrow \uparrow)$ DW and up-to-down $(\uparrow \nearrow \rightarrow \searrow \downarrow)$ DW, respectively. These features can be more clearly seen from the linescans shown in Fig. 5(d), which confirms that the spin chirality in the $\left[\mathrm{Mn}_{3} \mathrm{Sn} / \mathrm{Co}_{75} \mathrm{~Tb}_{25} / \mathrm{Si}_{3} \mathrm{~N}_{4}\right]_{20}$ multilayer is right handed. Experimental efforts are also made to directly image the spin chirality of Co at the $L_{3}$ edge, which is, however, unsuccessful due to a relatively smaller magnetization and hence a weaker XMCD-PEEM contrast. As a result of AFM coupling between the $\mathrm{Tb}$ and Co sublattices, the Co sublattice possesses the same spin chirality as the Tb sublattice, as schematically illustrated in the inset of Fig. 5(d).

\section{CONCLUSIONS}

In summary, using the noncollinear $\mathrm{AFM} \mathrm{Mn}_{3} \mathrm{Sn}$ as a seeding layer and the accompanied interfacial AFM exchange coupling through interfacing with a thin FIM $\mathrm{CoTb}$ layer, we have stabilized FIM Néel skyrmions in $\left[\mathrm{Mn}_{3} \mathrm{Sn} / \mathrm{Co}_{75} \mathrm{~Tb}_{25} / \mathrm{Si}_{3} \mathrm{~N}_{4}\right]_{20}$ and $\left[\mathrm{Si}_{3} \mathrm{~N}_{4} / \mathrm{Co}_{75} \mathrm{~Tb}_{25} / \mathrm{Mn}_{3} \mathrm{Sn}\right]_{20}$ multilayers, respectively. The opposite (right/left handed) spin chirality due to the flipped interfaces and hence the opposite (positive/negative) sign of the iDMI can be directly identified by using the 4D LSTEM in combination with imaging simulations. The 4D LSTEM results, together with the FIM nature of the Néel skyrmions in our multilayers, are further confirmed using XMCD-PEEM. Our results suggest that the exchange coupling between a noncollinear AFM and a thin FIM could similarly host an iDMI of strength $1.5 \pm 0.1 \mathrm{~mJ} / \mathrm{m}^{2}$ that can be harvested to stabilize room-temperature Néel skyrmions. Meanwhile, it is interesting to note that a negative sign of the iDMI parameter has been measured in $\mathrm{IrMn} / \mathrm{CoFeB} / \mathrm{MgO}$ trilayers $[19,73,74]$, as compared with the positive sign of the iDMI in the $\mathrm{Mn}_{3} \mathrm{Sn} / \mathrm{Co}_{75} \mathrm{~Tb}_{25} / \mathrm{Si}_{3} \mathrm{~N}_{4}$ multilayer. Thus, a precise understanding of the rich interfacial chiral magnetism in similar AFM-based quantum materials, however, requires further theoretical investigations, ideally from first-principles and $a b$ initio calculations [75]. We also summarized different skyrmion hosting materials in a table in Part 12 of the Supplemental Material [47], which could be beneficial for future material optimization. Compared with the other materials, the choice of FIM CoTb with a bulk PMA could stimulate more applications of quantum materials in chiral magnetism. Furthermore, one could envision harvesting the accompanied magnetic spin Hall effects from the topological $\mathrm{Mn}_{3} \mathrm{Sn}$ to study the current-driven dynamics of FIM Néel skyrmions [52,59,76], which may simultaneously reveal interesting physics such as the reduced skyrmion Hall effect. Meanwhile, one could explore the interlayer exchange between the skyrmion hosting multilayers and $\mathrm{Mn}_{3} \mathrm{Sn}$, for stabilizing AFM skyrmions. It is expected that this paper could trigger more investigations for gaining a comprehensive understanding of chiral interfacial magnetism and, meanwhile, could set a valuable step for bridging AFM spintronics and skyrmionics in the future $[77,78]$.

\section{ACKNOWLEDGMENTS}

Work carried out at Tsinghua was supported by the National Key R\&D Program of China (Grant No. 2017YFA0206200), Basic Science Center Project of the National Natural Science Foundation of China (NSFC; Grant No. 51788104), National Key R\&D Program of China (Grant No. 2016YFA0302300), the NSFC (Grants No. 11774194, No. 51831005, No. 1181101082, and No. 11804182), Beijing Natural Science Foundation (Grant No. Z190009), Tsinghua University Initiative Scientific Research Program, and the Beijing Advanced Innovation Center for Future Chip (ICFC). Electron microscopy at Cornell University was supported by U.S. Defense Advanced Research Projects Agency (TEE-D18AC00009) and U.S. Platform for the Accelerated Realization, Analysis, and Discovery of Interface Materials National Science Foundation (NSF) Materials Innovation Platform (No. DMR-1539918). Electron microscopy facilities at Cornell are supported by the Cornell Center for Materials Research, NSF Materials Research Science and Engineering Centers (No. DMR-1719875). Work at the ALS were supported by the U.S. Department of Energy (No. DE-AC02-05CH11231). Part of this paper was carried out at the Surface/Interface: Microscopy beamline of the Swiss Light Source. Funding by the Swiss National Science Foundation through Grant No. 200021160186 and Swiss Nanoscience Institute through Grant No. P1502 is acknowledged. M.-Y.I. acknowledges support by Lawrence Berkeley National Laboratory through the Laboratory Directed Research and Development (LDRD) Program and the National Research Foundation of Korea (NRF) grant funded by the Korea government (MSIT; No. NRF2019R1A2C2002996, No. NRF-2016M3D1A1027831, and No. NRF-2019K1A3A7A09033400). R.T. and G.F. thank the project "ThunderSKY" funded from the Hellenic Foundation for Research and Innovation (HFRI) and the General Secretariat for Research and Technology (GSRT) under Grant No. 871 . 
[1] U. K. Rößler, A. N. Bogdanov, and C. Pfleiderer, Spontaneous skyrmion ground states in magnetic metals, Nature 442, 797 (2006).

[2] S. Mühlbauer, B. Binz, F. Jonietz, C. Pfleiderer, A. Rosch, A. Neubauer, R. Georgii, and P. Böni, Skyrmion lattice in a chiral magnet, Science 323, 915 (2009).

[3] X. Z. Yu, Y. Onose, N. Kanazawa, J. H. Park, J. H. Han, Y. Matsui, N. Nagaosa, and Y. Tokura, Real-space observation of a two-dimensional skyrmion crystal, Nature 465, 901 (2010).

[4] S. Heinze, K. von Bergmann, M. Menzel, J. Brede, A. Kubetzka, R. Wiesendanger, G. Bihlmayer, and S. Blügel, Spontaneous atomic-scale magnetic skyrmion lattice in two dimensions, Nat. Phys. 7, 713 (2011).

[5] N. Romming, C. Hanneken, M. Menzel, J. E. Bickel, B. Wolter, K. von Bergmann, A. Kubetzka, and R. Wiesendanger, Writing and deleting single magnetic skyrmions, Science 341, 636 (2013).

[6] W. Jiang, P. Upadhyaya, W. Zhang, G. Yu, M. B. Jungfleisch, F. Y. Fradin, J. E. Pearson, Y. Tserkovnyak, K. L. Wang, O. Heinonen, S. G. E. te Velthuis, and A. Hoffmann, Blowing magnetic skyrmion bubbles, Science 349, 283 (2015).

[7] C. Moreau-Luchaire, C. Moutafis, N. Reyren, J. Sampaio, C. A. F. Vaz, N. Van Horne, K. Bouzehouane, K. Garcia, C. Deranlot, P. Warnicke, P. Wohlhüter, J. M. George, M. Weigand, J. Raabe, V. Cros, and A. Fert, Additive interfacial chiral interaction in multilayers for stabilization of small individual skyrmions at room temperature, Nat. Nanotechnol. 11, 444 (2016).

[8] S. Woo, K. Litzius, B. Krüger, M.-Y. Im, L. Caretta, K. Richter, M. Mann, A. Krone, R. M. Reeve, M. Weigand, P. Agrawal, I. Lemesh, M.-A. Mawass, P. Fischer, M. Kläui, and G. S. D. Beach, Observation of room-temperature magnetic skyrmions and their current-driven dynamics in ultrathin metallic ferromagnets, Nat. Mater. 15, 501 (2016).

[9] A. Fert, N. Reyren, and V. Cros, Magnetic skyrmions: advances in physics and potential applications, Nat. Rev. Mater. 2, 17031 (2017).

[10] W. Jiang, G. Chen, K. Liu, J. Zang, S. G. E. te Velthuis, and A. Hoffmann, Skyrmions in magnetic multilayers, Phys. Rep. 704, 1 (2017).

[11] T. Yokouchi, S. Sugimoto, B. Rana, S. Seki, N. Ogawa, S. Kasai, and Y. Otani, Creation of magnetic skyrmions by surface acoustic waves, Nat. Nanotechnol. 15, 361 (2020).

[12] S. Woo, K. M. Song, X. Zhang, Y. Zhou, M. Ezawa, X. Liu, S. Finizio, J. Raabe, N. J. Lee, S.-I. Kim, S.-Y. Park, Y. Kim, J.-Y. Kim, D. Lee, O. Lee, J. W. Choi, B.-C. Min, H. C. Koo, and J. Chang, Current-driven dynamics and inhibition of the skyrmion Hall effect of ferrimagnetic skyrmions in GdFeCo films, Nat. Commun. 9, 959 (2018).

[13] S. D. Pollard, J. A. Garlow, J. Yu, Z. Wang, Y. Zhu, and H. Yang, Observation of stable Néel skyrmions in cobalt/palladium multilayers with Lorentz transmission electron microscopy, Nat. Commun. 8, 14761 (2017).

[14] S. McVitie, S. Hughes, K. Fallon, S. McFadzean, D. McGrouther, M. Krajnak, W. Legrand, D. Maccariello, S. Collin, K. Garcia, N. Reyren, V. Cros, A. Fert, K. Zeissler, and C. H. Marrows, A transmission electron microscope study of Néel skyrmion magnetic textures in multilayer thin film systems with large interfacial chiral interaction, Sci. Rep. 8, 5703 (2018).
[15] W. Jiang, X. Zhang, G. Yu, W. Zhang, X. Wang, M. Benjamin Jungfleisch, J. E. Pearson, X. Cheng, O. Heinonen, K. L. Wang, Y. Zhou, A. Hoffmann, and S. G. E. t. Velthuis, Direct observation of the skyrmion Hall effect, Nat. Phys. 13, 162 (2017).

[16] Z. Wang, M. Guo, H.-A. Zhou, L. Zhao, T. Xu, R. Tomasello, H Bai, Y. Dong, S.-G. Je, W. Chao, H.-S. Han, S. Lee, K.-S. Lee, Y. Yao, W. Han, C. Song, H. Wu, M. Carpentieri, G. Finocchio, M.-Y. Im, S.-Z. Lin, and W. Jiang, Thermal generation, manipulation and thermoelectric detection of skyrmions, Nat. Electron. 3, 672 (2020).

[17] S. Jaiswal, K. Litzius, I. Lemesh, F. Büttner, S. Finizio, J. Raabe, M. Weigand, K. Lee, J. Langer, B. Ocker, G. Jakob, G. S. D. Beach, and M. Kläui, Investigation of the Dzyaloshinskii-Moriya interaction and room temperature skyrmions in $\mathrm{W} / \mathrm{CoFeB} / \mathrm{MgO}$ thin films and microwires, Appl. Phys. Lett. 111, 022409 (2017).

[18] T. Nozaki, Y. Jibiki, M. Goto, E. Tamura, T. Nozaki, H. Kubota, A. Fukushima, S. Yuasa, and Y. Suzuki, Brownian motion of skyrmion bubbles and its control by voltage applications, Appl. Phys. Lett. 114, 012402 (2019).

[19] G. Yu, A. Jenkins, X. Ma, S. A. Razavi, C. He, G. Yin, Q. Shao, Q. 1. He, H. Wu, W. Li, W. Jiang, X. Han, X. Li, A. C. Bleszynski Jayich, P. K. Amiri, and K. L. Wang, Room-temperature skyrmions in an antiferromagnet-based heterostructure, Nano Lett. 18, 980 (2018).

[20] M. Bode, M. Heide, K. von Bergmann, P. Ferriani, S. Heinze, G. Bihlmayer, A. Kubetzka, O. Pietzsch, S. Blügel, and R. Wiesendanger, Chiral magnetic order at surfaces driven by inversion asymmetry, Nature 447, 190 (2007).

[21] M. Heide, G. Bihlmayer, and S. Blügel, Dzyaloshinskii-Moriya interaction accounting for the orientation of magnetic domains in ultrathin films: Fe/W(110), Phys. Rev. B 78, 140403(R) (2008).

[22] G. Chen, J. Zhu, A. Quesada, J. Li, A. T. N'Diaye, Y. Huo, T. P. Ma, Y. Chen, H. Y. Kwon, C. Won, Z. Q. Qiu, A. K. Schmid, and Y. Z. Wu, Novel Chiral Magnetic Domain Wall Structure in $\mathrm{Fe} / \mathrm{Ni} / \mathrm{Cu}(001)$ Films, Phys. Rev. Lett. 110, 177204 (2013).

[23] G. Chen, A. Mascaraque, A. T. N'Diaye, and A. K. Schmid, Room temperature skyrmion ground state stabilized through interlayer exchange coupling, Appl. Phys. Lett. 106, 242404 (2015).

[24] O. Boulle, J. Vogel, H. Yang, S. Pizzini, D. de Souza Chaves, A. Locatelli, T. O. Menteş, A. Sala, L. D. Buda-Prejbeanu, O. Klein, M. Belmeguenai, Y. Roussigné, A. Stashkevich, S. M. Chérif, L. Aballe, M. Foerster, M. Chshiev, S. Auffret, I. M. Miron, and G. Gaudin, Room-temperature chiral magnetic skyrmions in ultrathin magnetic nanostructures, Nat. Nanotechnol. 11, 449 (2016).

[25] A. Soumyanarayanan, M. Raju, A. L. Gonzalez Oyarce, A. K. C. Tan, M.-Y. Im, A. P. Petrović, P. Ho, K. H. Khoo, M. Tran, C. K. Gan, F. Ernult, and C. Panagopoulos, Tunable room-temperature magnetic skyrmions in $\mathrm{Ir} / \mathrm{Fe} / \mathrm{Co} / \mathrm{Pt}$ multilayers, Nat. Mater. 16, 898 (2017).

[26] H. Wu, F. Groß, B. Dai, D. Lujan, S. A. Razavi, P. Zhang, Y. Liu, K. Sobotkiewich, J. Förster, M. Weigand, G. Schütz, X. Li, J. Gräfe, and K. L. Wang, Ferrimagnetic skyrmions in topological insulator/ferrimagnet heterostructures, Adv. Mater. 32, 2003380 (2020).

[27] G. Chen, T. Ma, A. T. N'Diaye, H. Kwon, C. Won, Y. $\mathrm{Wu}$, and A. K. Schmid, Tailoring the chirality of magnetic 
domain walls by interface engineering, Nat. Commun. 4, 2671 (2013).

[28] K. Zeissler, S. Finizio, K. Shahbazi, J. Massey, F. A. Ma’Mari, D. M. Bracher, A. Kleibert, M. C. Rosamond, E. H. Linfield, T. A. Moore, J. Raabe, G. Burnell, and C. H. Marrows, Discrete Hall resistivity contribution from Néel skyrmions in multilayer nanodiscs, Nat. Nanotechnol. 13, 1161 (2018).

[29] A. Soumyanarayanan, N. Reyren, A. Fert, and C. Panagopoulos, Emergent phenomena induced by spin-orbit coupling at surfaces and interfaces, Nature 539, 509 (2016).

[30] F. Hellman et al., Interface-induced phenomena in magnetism, Rev. Mod. Phys. 89, 025006 (2017).

[31] N. Nagaosa and Y. Tokura, Topological properties and dynamics of magnetic skyrmions, Nat. Nanotechnol. 8, 899 (2013).

[32] M. J. Benitez, A. Hrabec, A. P. Mihai, T. A. Moore, G. Burnell, D. McGrouther, C. H. Marrows, and S. McVitie, Magnetic microscopy and topological stability of homochiral Néel domain walls in a Pt/Co/AlOx trilayer, Nat. Commun. 6, 8957 (2015).

[33] W. Jiang, S. Zhang, X. Wang, C. Phatak, Q. Wang, W. Zhang, M. B. Jungfleisch, J. E. Pearson, Y. Liu, J. Zang, X. Cheng, A. Petford-Long, A. Hoffmann, and S. G. E. te Velthuis, Quantifying chiral exchange interaction for Neel-type skyrmions via Lorentz transmission electron microscopy, Phys. Rev. B 99, 104402 (2019).

[34] A. Fert, V. Cros, and J. Sampaio, Skyrmions on the track, Nat. Nanotechnol. 8, 152 (2013).

[35] J. Sampaio, V. Cros, S. Rohart, A. Thiaville, and A. Fert, Nucleation, stability and current-induced motion of isolated magnetic skyrmions in nanostructures, Nat. Nanotechnol. 8, 839 (2013).

[36] X. Yu, M. Mostovoy, Y. Tokunaga, W. Zhang, K. Kimoto, Y. Matsui, Y. Kaneko, N. Nagaosa, and Y. Tokura, Magnetic stripes and skyrmions with helicity reversals, Proc. Natl. Acad. Sci. 109, 8856 (2012).

[37] S. A. Montoya, S. Couture, J. J. Chess, J. C. T. Lee, N. Kent, D. Henze, S. K. Sinha, M. Y. Im, S. D. Kevan, P. Fischer, B. J. McMorran, V. Lomakin, S. Roy, and E. E. Fullerton, Tailoring magnetic energies to form dipole skyrmions and skyrmion lattices, Phys. Rev. B 95, 024415 (2017).

[38] J. C. Loudon, A. C. Twitchett-Harrison, D. Cortés-Ortuño, M. T. Birch, L. A. Turnbull, A. Štefančič, F. Y. Ogrin, E. O. Burgos-Parra, N. Bukin, A. Laurenson, H. Popescu, M. Beg, O. Hovorka, H. Fangohr, P. A. Midgley, G. Balakrishnan, and P. D. Hatton, Do images of biskyrmions show type-II bubbles? Adv. Mater. 31, 1806598 (2019).

[39] S. McVitie and M. Cushley, Quantitative Fresnel Lorentz microscopy and the transport of intensity equation, Ultramicroscopy 106, 423 (2006).

[40] M. Krajnak, D. McGrouther, D. Maneuski, V. O. Shea, and S. McVitie, Pixelated detectors and improved efficiency for magnetic imaging in STEM differential phase contrast, Ultramicroscopy 165, 42 (2016).

[41] M. He, L. Peng, Z. Zhu, G. Li, J. Cai, J. Li, H. Wei, L. Gu, S. Wang, T. Zhao, B. Shen, and Y. Zhang, Realization of zero-field skyrmions with high-density via electromagnetic manipulation in Pt/Co/Ta multilayers, Appl. Phys. Lett. 111, 202403 (2017).

[42] K. X. Nguyen, E. Turgut, M. C. Cao, J. Glaser, Z. Chen, M. J. Stolt, S. Jin, G. D. Fuchs, and D. A. Muller, Disentangling magnetic and grain contrast in polycrystalline FeGe thin films using 4-D Lorentz Scanning Transmission Electron Microscopy, arXiv:2001.06900 (2020).
[43] N. Romming, A. Kubetzka, C. Hanneken, K. von Bergmann, and R. Wiesendanger, Field-Dependent Size and Shape of Single Magnetic Skyrmions, Phys. Rev. Lett. 114, 177203 (2015).

[44] J. Lucassen, M. J. Meijer, O. Kurnosikov, H. J. M. Swagten, B. Koopmans, R. Lavrijsen, F. Kloodt-Twesten, R. Frömter, and R. A. Duine, Tuning Magnetic Chirality by Dipolar Interactions, Phys. Rev. Lett. 123, 157201 (2019).

[45] K. v. Bergmann, A. Kubetzka, O. Pietzsch, and R. Wiesendanger, Interface-induced chiral domain walls, spin spirals and skyrmions revealed by spin-polarized scanning tunneling microscopy, J. Phys.: Condens. Matter 26, 394002 (2014).

[46] M. J. Meijer, J. Lucassen, F. Kloodt-Twesten, R. Frömter, O. Kurnosikov, R. A. Duine, H. J. M. Swagten, B. Koopmans, and R. Lavrijsen, Magnetic Chirality Controlled by the Interlayer Exchange Interaction, Phys. Rev. Lett. 124, 207203 (2020).

[47] See Supplemental Material at http://link.aps.org/supplemental/ 10.1103/PhysRevMaterials.5.084406 for additional information on technique and material comparisons, cross-sectional layer-structures, MTXM and LTEM images, micromagnetic simulation, magnetic flux from in-plane $\left(m_{x, y}\right)$ and out-of-plane $\left(m_{z}\right)$ components, simulated and experimental vertical component $B_{y}$ and the magnitude $|B|$, and XAS and XMCD spectra at the $\mathrm{Mn} L_{2,3}$ edge.

[48] T. Nagamiya, Triangular spin ordering in $\mathrm{Mn}_{3} \mathrm{Sn}$ and $\mathrm{Mn}_{3} \mathrm{Ge}$, J. Phys. Soc. Jpn. 46, 787 (1979).

[49] S. Nakatsuji, N. Kiyohara, and T. Higo, Large anomalous Hall effect in a non-collinear antiferromagnet at room temperature, Nature 527, 212 (2015).

[50] M. Ikhlas, T. Tomita, T. Koretsune, M.-T. Suzuki, D. NishioHamane, R. Arita, Y. Otani, and S. Nakatsuji, Large anomalous Nernst effect at room temperature in a chiral antiferromagnet, Nat. Phys. 13, 1085 (2017).

[51] T. Higo, H. Man, D. B. Gopman, L. Wu, T. Koretsune, O. M. J. van 't Erve, Y. P. Kabanov, D. Rees, Y. Li, M.-T. Suzuki, S. Patankar, M. Ikhlas, C. L. Chien, R. Arita, R. D. Shull, J. Orenstein, and S. Nakatsuji, Large magneto-optical Kerr effect and imaging of magnetic octupole domains in an antiferromagnetic metal, Nat. Photonics 12, 73 (2018).

[52] M. Kimata, H. Chen, K. Kondou, S. Sugimoto, P. K. Muduli, M. Ikhlas, Y. Omori, T. Tomita, A. H. MacDonald, S. Nakatsuji, and Y. Otani, Magnetic and magnetic inverse spin Hall effects in a non-collinear antiferromagnet, Nature 565, 627 (2019).

[53] K. Kuroda et al., Evidence for magnetic Weyl fermions in a correlated metal, Nat. Mater. 16, 1090 (2017).

[54] Y. Zhang, J. Železný, Y. Sun, J. van den Brink, and B. Yan, Spin Hall effect emerging from a noncollinear magnetic lattice without spin-orbit coupling, New J. Phys. 20, 073028 (2018).

[55] X. Li, C. Collignon, L. Xu, H. Zuo, A. Cavanna, U. Gennser, D. Mailly, B. Fauqué, L. Balents, Z. Zhu, and K. Behnia, Chiral domain walls of $\mathrm{Mn}_{3} \mathrm{Sn}$ and their memory, Nat. Commun. 10, 3021 (2019).

[56] H. Yang, Y. Sun, Y. Zhang, W.-J. Shi, S. S. P. Parkin, and B. Yan, Topological Weyl semimetals in the chiral antiferromagnetic materials $\mathrm{Mn}_{3}$ Ge and $\mathrm{Mn}_{3} \mathrm{Sn}$, New J. Phys. 19, 015008 (2017).

[57] R. Cardias, A. Bergman, A. Szilva, Y. O. Kvashnin, J. Fransson, A. B. Klautau, O. Eriksson, and L. Nordström, Dzyaloshinskii-Moriya interaction in absence of spin-orbit coupling, arXiv:2003.04680 (2020). 
[58] R. Yanes, J. Jackson, L. Udvardi, L. Szunyogh, and U. Nowak, Exchange Bias Driven by Dzyaloshinskii-Moriya Interactions, Phys. Rev. Lett. 111, 217202 (2013).

[59] Y. Zhang, Y. Sun, H. Yang, J. Železný, S. P. P. Parkin, C. Felser, and B. Yan, Strong anisotropic anomalous Hall effect and spin Hall effect in the chiral antiferromagnetic compounds $\mathrm{Mn}_{3} X$ ( $X=\mathrm{Ge}, \mathrm{Sn}, \mathrm{Ga}$, Ir, Rh, and Pt), Phys. Rev. B 95, 075128 (2017).

[60] S. Tomiyoshi and Y. Yamaguchi, Magnetic structure and weak ferromagnetism of $\mathrm{Mn}_{3} \mathrm{Sn}$ studied by polarized neutron diffraction, J. Phys. Soc. Jpn. 51, 2478 (1982).

[61] P. J. Brown, V. Nunez, F. Tasset, J. B. Forsyth, and P. Radhakrishna, Determination of the magnetic structure of $\mathrm{Mn}_{3} \mathrm{Sn}$ using generalized neutron polarization analysis, J. Phys.: Condens. Matter 2, 9409 (1990).

[62] J. Finley and L. Liu, Spin-Orbit-Torque Efficiency in Compensated Ferrimagnetic Cobalt-Terbium Alloys, Phys. Rev. Appl. 6, 054001 (2016).

[63] V. Puliafito, R. Khymyn, M. Carpentieri, B. Azzerboni, V. Tiberkevich, A. Slavin, and G. Finocchio, Micromagnetic modeling of terahertz oscillations in an antiferromagnetic material driven by the spin Hall effect, Phys. Rev. B 99, 024405 (2019).

[64] L. Sánchez-Tejerina, V. Puliafito, P. Khalili Amiri, M. Carpentieri, and G. Finocchio, Dynamics of domain-wall motion driven by spin-orbit torque in antiferromagnets, Phys. Rev. B 101, 014433 (2020).

[65] M. Binder, A. Weber, O. Mosendz, G. Woltersdorf, M. Izquierdo, I. Neudecker, J. R. Dahn, T. D. Hatchard, J. U. Thiele, C. H. Back, and M. R. Scheinfein, Magnetization dynamics of the ferrimagnet CoGd near the compensation of magnetization and angular momentum, Phys. Rev. B 74, 134404 (2006).

[66] Y. Ogata, H. Chudo, M. Ono, K. Harii, M. Matsuo, S. Maekawa, and E. Saitoh, Gyroscopic $g$ factor of rare earth metals, Appl. Phys. Lett. 110, 072409 (2017).

[67] S. A. Siddiqui, J. Han, J. T. Finley, C. A. Ross, and L. Liu, Current-Induced Domain Wall Motion in a Compensated Ferrimagnet, Phys. Rev. Lett. 121, 057701 (2018).

[68] S. McVitie, D. McGrouther, S. McFadzean, D. A. MacLaren, K. J. O'Shea, and M. J. Benitez, Aberration corrected Lorentz scanning transmission electron microscopy, Ultramicroscopy 152, 57 (2015)

[69] M. Mansuripur, Computation of electron-diffraction patterns in Lorentz electron microscopy of thin magnetic films, J. Appl. Phys. 69, 5890 (1991).

[70] W. Legrand, J.-Y. Chauleau, D. Maccariello, N. Reyren, S Collin, K. Bouzehouane, N. Jaouen, V. Cros, and A. Fert, Hybrid chiral domain walls and skyrmions in magnetic multilayers, Sci. Adv. 4, eaat0415 (2018).

[71] J. N. Chapman, P. E. Batson, E. M. Waddell, and R. P. Ferrier, The direct determination of magnetic domain wall profiles by differential phase contrast electron microscopy, Ultramicroscopy 3, 203 (1978).

[72] J. Zhang, X. Zhang, H. Chen, Y. Guang, X. Zeng, G. Yu, S. Zhang, Y. Liu, J. Feng, Y. Zhao, Y. Zhou, X. Qiu, X. Han, Y. Peng, and $X$. Zhang, Formation and magnetic-field stability of magnetic dipole skyrmions and bubbles in a ferrimagnet, Appl. Phys. Lett. 116, 142404 (2020).

[73] X. Ma, G. Yu, S. A. Razavi, S. S. Sasaki, X. Li, K. Hao, S. H. Tolbert, K. L. Wang, and X. Li, Dzyaloshinskii-Moriya Interaction across an Antiferromagnet-Ferromagnet Interface, Phys. Rev. Lett. 119, 027202 (2017).

[74] Y. Guang, I. Bykova, Y. Liu, G. Yu, E. Goering, M. Weigand, J. Gräfe, S. K. Kim, J. Zhang, H. Zhang, Z. Yan, C. Wan, J. Feng, X. Wang, C. Guo, H. Wei, Y. Peng, Y. Tserkovnyak, X. Han, and G. Schütz, Creating zero-field skyrmions in exchangebiased multilayers through $\mathrm{x}$-ray illumination, Nat. Commun. 11, 949 (2020).

[75] H. Yang, A. Thiaville, S. Rohart, A. Fert, and M. Chshiev, Anatomy of Dzyaloshinskii-Moriya Interaction at $\mathrm{Co} / \mathrm{Pt}$ Interfaces, Phys. Rev. Lett. 115, 267210 (2015).

[76] H. Tsai, T. Higo, K. Kondou, T. Nomoto, A. Sakai, A. Kobayashi, T. Nakano, K. Yakushiji, R. Arita, S. Miwa, Y. Otani, and S. Nakatsuji, Electrical manipulation of a topological antiferromagnetic state, Nature 580, 608 (2020).

[77] O. Gomonay, V. Baltz, A. Brataas, and Y. Tserkovnyak, Antiferromagnetic spin textures and dynamics, Nat. Phys. 14, 213 (2018).

[78] T. Jungwirth, X. Marti, P. Wadley, and J. Wunderlich, Antiferromagnetic spintronics, Nat. Nanotechnol. 11, 231 (2016). 